\title{
Integrating gaze direction and sexual dimorphism of face shape when perceiving the dominance of others
}

\author{
Julie C Main, Benedict C Jones $\uparrow$, Lisa M DeBruine \\ School of Psychology, University of Aberdeen, Regent Walk, Aberdeen AB9 1FX, Scotland, UK; \\ e-mail: ben.jones@abdn.ac.uk
}

\section{Anthony C Little}

Department of Psychology, University of Stirling, Stirling FK9 4LA, Scotland, UK

Received 8 December 2008, in revised form 13 March 2009

\begin{abstract}
Although gaze direction and face shape have each been shown to affect perceptions of the dominance of others, the question whether gaze direction and face shape have independent main effects on perceptions of dominance, and whether these effects interact, has not yet been studied. To investigate this issue, we compared dominance ratings of faces with masculinised shapes and direct gaze, masculinised shapes and averted gaze, feminised shapes and direct gaze, and feminised shapes and averted gaze. While faces with direct gaze were generally rated as more dominant than those with averted gaze, this effect of gaze direction was greater when judging faces with masculinised shapes than when judging faces with feminised shapes. Additionally, faces with masculinised shapes were rated as more dominant than those with feminised shapes when faces were presented with direct gaze, but not when faces were presented with averted gaze. Collectively, these findings reveal an interaction between the effects of gaze direction and sexually dimorphic facial cues on judgments of the dominance of others, presenting novel evidence for the existence of complex integrative processes that underpin social perception of faces. Integrating information from face shape and gaze cues may increase the efficiency with which we perceive the dominance of others.
\end{abstract}

\section{Introduction}

The majority of studies of facial attractiveness have emphasised the importance of physical characteristics, such as sexual dimorphism of face shape, symmetry and averageness (see Fink and Penton-Voak 2002 and Rhodes 2006 for reviews). More recent studies, however, have demonstrated that facial attractiveness is influenced by interactions between relatively invariant physical cues in faces and more changeable social signals, such as gaze direction (Conway et al 2008a, 2008b; Jones et al 2006; Kampe et al 2001). For example, Jones et al (2006) found that preferences for attractive colour and texture cues were stronger when judging the attractiveness of faces that were smiling at the viewer than when judging faces that were looking at the viewer with a neutral expression or that were looking away from the viewer. Additionally, neurobiological evidence suggests that direct gaze increases the reward value of attractive faces (ie the extent to which viewing attractive faces causes activity in brain regions associated with processing rewards), but not of relatively unattractive faces (Kampe et al 2001). Other recent studies have shown that perceiver-directed smiles are considered more attractive from opposite-sex individuals than own-sex individuals (Conway et al 2008a) and from healthy-looking, attractive individuals than relatively unhealthy-looking, unattractive individuals (Conway et al 2008b). While these studies demonstrate interactions between gaze direction and physical characteristics in faces when perceiving the attractiveness of others, it is not known whether similar interactions occur for other types of social attribution, such as dominance.

Perceptions of the dominance of others affect social behaviour in many primate species, including humans (Emery 2000; Oosterhof and Todorov 2008; Perrett et al 1998; 
Puts et al 2006). Indeed, Oosterhof and Todorov (2008) recently identified perceptions of dominance as a particularly important determinant of social perception of faces. In humans, sexual dimorphism of two-dimensional (2-D) face shape affects attributions of dominance, with masculine faces being perceived as more dominant than feminine faces (DeBruine et al 2006; Perrett et al 1998). Indeed, measures of upper-body strength are positively associated with both dominance and masculinity ratings of faces, suggesting that perceptions of the dominance of others from facial cues may reflect perceptions of their ability to physically dominate others (Fink et al 2007). The association between masculine traits and perceived dominance is not limited to perceptions of faces and has also been observed in other domains (eg perceptions of voices-Feinberg et al 2005; Puts et al 2006). While these studies suggest that dominance is associated with masculine physical characteristics, social signals (eg gaze direction) also affect perceptions of dominance. For example, direct gaze increases the perceived dominance of individuals (see eg Burgoon et al 1986) and also increases attributions of anger to faces (Adams and Kleck 2005). It is unclear, however, whether gaze direction and sexual dimorphism of 2-D face shape have only independent main effects on perceptions of dominance or whether these characteristics interact when judging the dominance of others. Integrating information from multiple cues of dominance (eg face shape, gaze direction) may function to increase the efficiency with which one attributes dominance to others during social interactions.

Adams and Kleck (2003) have previously found that viewers were more sensitive to angry facial expressions shown with direct gaze than angry facial expressions shown with averted gaze, but also found that viewers were more sensitive to fearful expressions shown with averted gaze than fearful expressions shown with direct gaze (but see also Bindemann et al 2008). That angry faces are rated as more dominant than fearful faces (Hess et al 2000) therefore raises the possibility that viewers may also be more sensitive to physical cues of dominance (eg masculine face shape) when judging the dominance of faces with direct gaze than when judging the dominance of faces with averted gaze. Furthermore, Adams and Kleck (2003) proposed that greater sensitivity to perceiver-directed anger than perceiver-directed fear may occur, at least partly, because direct gaze signals greater threat when shown in combination with an angry facial expression than when shown in combination with a fearful facial expression. This proposal raises the possibility that viewers may also be more sensitive to gaze direction when judging the dominance of masculinised (ie high-dominance) faces than when judging feminised (ie low-dominance) faces. In light of these possibilities, we tested for an interaction between gaze direction and sexually dimorphic shape characteristics when rating facial dominance. We tested two specific predictions: (i) that the effect of masculine facial characteristics on dominance ratings may be greater when rating faces that are directing their attention at the viewer (ie faces shown with direct gaze) than when rating faces that are directing their attention away from the viewer (ie faces with averted gaze) and (ii) that the effect of gaze direction on dominance ratings may be greater when rating masculinised faces than when rating feminised faces.

\section{Methods}

\subsection{Stimuli}

First, we manufactured male and female prototype face images with direct and averted gaze. The direct-gaze male prototype was manufactured by averaging the shape, colour, and texture information from full-face photographs of 24 white men (age: mean $=20.52$ years, $S D=4.57$ years; all undergraduate students at the University of Aberdeen) shown with direct gaze. The averted-gaze male prototype was manufactured by averaging the shape, colour, and texture information from full-face photographs 
of the same 24 men, this time with gaze averted to the left. Direct- and averted-gaze female prototypes were manufactured by using images of 24 white women (age: mean $=20.37$ years, $S D=5.24$ years; all undergraduate students at the University of Aberdeen) with direct- and averted-gaze, respectively. Technical details of the averaging procedure we used to manufacture these prototypes are given in Tiddeman et al (2001). These methods have been used to generate face stimuli in many previous studies (eg Conway et al 2008a, 2008b, 2009; DeBruine et al 2006; Jones et al 2004, 2005, 2006; Perrett et al 2002).

Following Jones et al (2006) and Conway et al (2009), we used the direct- and averted-gaze prototypes to alter the gaze direction of the direct-gaze prototypes, creating a version of each direct-gaze prototype in which gaze was averted to the left but in which all other aspects of the face were unaltered. We used versions of the direct-gaze prototypes in which gaze direction was averted to the left, rather than simply using the composite image manufactured from individual images with gaze averted to the left, to control for possible effects of aspects of facial appearance that may otherwise be correlated with averted gaze (eg subtle changes in head position). To manufacture a version of the male direct-gaze prototype in which gaze was averted to the left, we applied $100 \%$ of the linear differences between the $x, y$ coordinates defining the position of the irises in the averted-gaze and direct-gaze male (following Conway et al 2009 and Jones et al 2006; see Tiddeman et al 2001 for technical details of this linear-transformation method). These same methods were used to manufacture female prototypes with direct and averted gaze.

We next manufactured masculinised and feminised versions of the male and female direct-gaze prototypes and the versions in which gaze direction had been manipulated to show averted gaze. Masculinised and feminised versions were manufactured by adding or subtracting $50 \%$ of the linear differences in 2-D shape between symmetrised male and female prototypes with direct gaze. This method alters sexually dimorphic aspects of face shape (ie other aspects of facial appearance, such as gaze direction, skin colour, and skin texture, are unaltered), ensuring that masculinised and feminised prototypes with the same gaze direction and of the same sex differ in masculinity-femininity of face shape, but are matched in all other regards. Additionally, this method for masculinising and feminising face images ensures that the extent of masculinisation and feminisation is identical across different combinations of gaze direction and sex. In many previous studies these methods have been used to manufacture masculinised and feminised face images (Buckingham et al 2006; DeBruine et al 2006; Jones et al 2007; Little et al 2001, 2002; Penton-Voak et al 1999, 2003; Perrett et al 1998; Welling et al 2007, 2008). Masculinised faces manufactured by these methods are perceived as more masculine (see, eg, DeBruine et al 2006; Jones et al 2007; Perrett et al 1998; Welling et al 2007, 2008) and more dominant (DeBruine et al 2006; Perrett et al 1998) than their feminised counterparts. All images were cropped around the face, ears, and neck so that hairstyle and clothing were not visible (see figure 1).

In total, the process described above produced eight prototype faces representing all possible combinations of gaze direction (direct or averted), shape (masculinised or feminised), and sex (male or female) of face. Examples of these images are shown in figure 1 .

\subsection{Procedure}

Participants $(N=45$; age: mean $=23.91$ years, $\mathrm{SD}=9.14$ years; twenty-four female, twenty-one male) rated the faces for dominance using a 1 (not very dominant) to 7 (very dominant) scale. The order in which face images were presented was fully randomised for each participant. Each image was presented individually and was presented (and rated) on two separate occasions in the same block of trials. Each individual image remained 


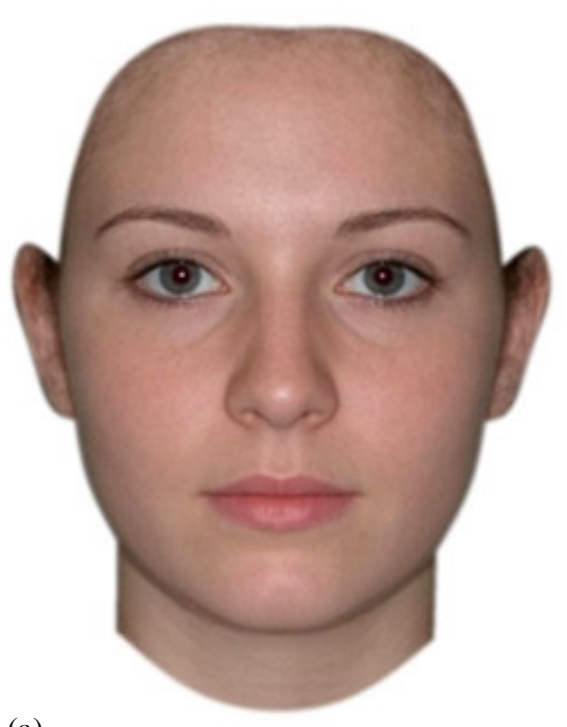

(a)

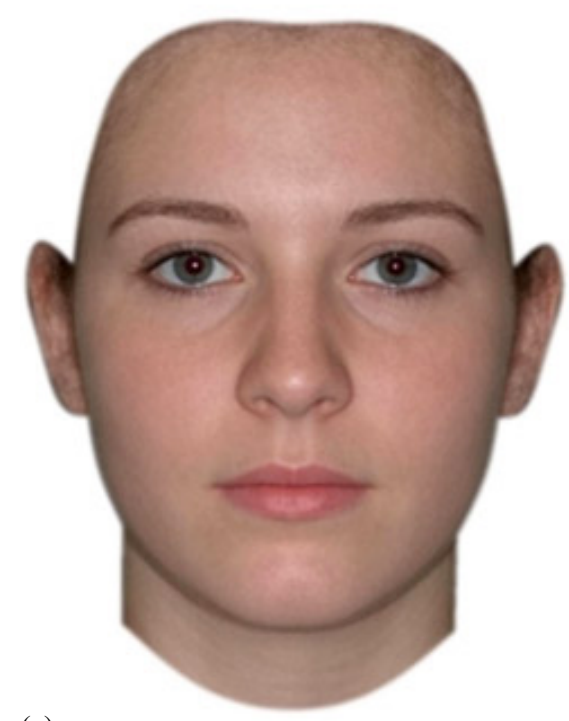

(c)

Figure 1. [In colour online, see http://dx.doi.org/10.1068/p6347] Feminised (a) and (b), and masculinised (c) and (d) faces. Faces with direct gaze (a) and (c), and averted gaze (b) and (d).

on screen until the participant had rated it, at which point the next image appeared. Participants were also asked to report their ethnicity (White: $N=35$; East Asian: $N=2$; West Asian: $N=5$; three participants elected not to report their ethnicity).

The experiment was run online with participants recruited from various online lists of web-based experiments (eg psychcentral.com). Participants were not compensated for participation and none of our participants were students or staff at the University of Aberdeen. Deaner et al (2007) have previously shown enhanced gaze-cuing effects when viewing familiar faces among women, but not among men. That our participants were not affiliated with the University of Aberdeen controls for such effects of familiarity with the individuals from which our face-stimuli were manufactured (all of whom 
were students at the University of Aberdeen). Following Kraut et al (2004), data from repeat IP addresses were not recorded, controlling for the possibility that some participants may have repeated the test. Many previous online studies of face perception have reported effects that are consistent with findings in lab-based studies (eg DeBruine et al 2007; Jones et al 2005, 2007; Wilson and Daly 2004). Indeed, previous studies have demonstrated identical effects of gaze direction in laboratory and online samples (eg Conway et al 2008a).

\section{Results}

Inter-rater agreement for dominance ratings was high (Cronbach's $\alpha=0.91$ ). For each participant, we calculated the mean dominance rating for each condition. Mean dominance ratings were normally distributed in each condition (Kolmogorov-Smirnov tests: all $z<1.10$, all $p>0.19$ ).

Dominance ratings were then analysed with a mixed-design ANOVA [within-subjects factors: sex of face (male, female), face shape (masculinised, feminised), gaze direction (direct, averted); between-subjects factor: sex of participant (male, female)]. This analysis revealed a significant main effect of sex of face $\left(F_{1,43}=20.77, p<0.001\right.$, partial $\left.\eta^{2}=0.33\right)$, whereby male faces were generally rated as more dominant than female faces (male faces: mean $=3.97, \mathrm{SEM}=0.16$; female faces: mean $=3.21, \mathrm{SEM}=0.16$ ). There was also a significant main effect of gaze direction $\left(F_{1,43}=30.74, p<0.001\right.$, partial $\eta^{2}=0.42$ ), whereby faces with direct gaze were generally rated as more dominant than faces with averted gaze (direct gaze: mean $=4.02, \mathrm{SEM}=0.16$; averted gaze: mean $=3.16, \mathrm{SEM}=0.15$ ). A main effect of face shape, whereby masculinised faces tended to be rated as more dominant than feminised faces (masculinised faces: mean $=3.67, \mathrm{SEM}=0.14$; feminised faces: mean $=3.51, \mathrm{SEM}=0.14$ ), approached significance $\left(F_{1,43}=3.81, p=0.058\right.$, partial $\left.\eta^{2}=0.08\right)$. As we predicted, there was a significant interaction between face shape and gaze direction $\left(F_{1,43}=6.34, p=0.016\right.$, partial $\eta^{2}=0.13$; see figure 2). There were no other significant effects (all $F<2.60$, all $p>0.11$, all partial $\eta^{2}<0.06$ ).

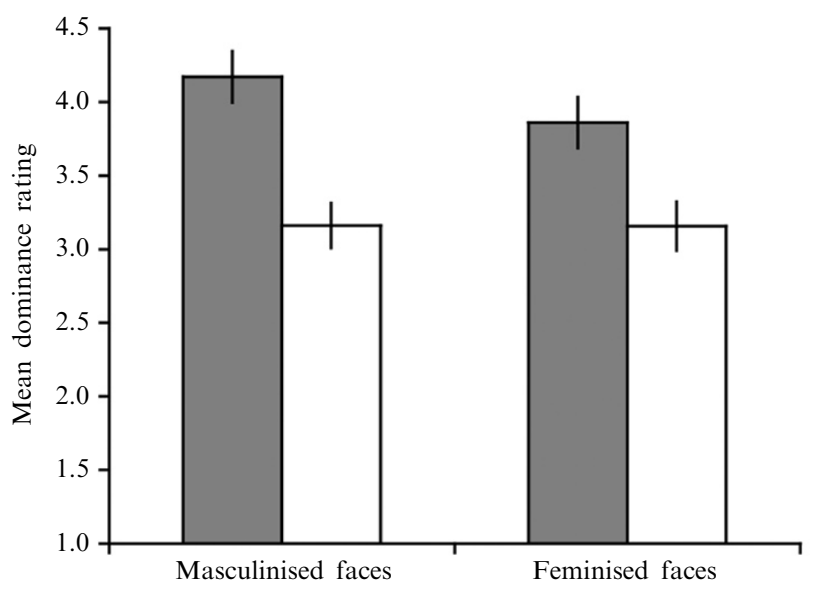

direct gaze

averted gaze

Figure 2. The significant interaction between face shape and gaze direction. Bars show means and SEMs.

Next, we carried out tests for simple effects to interpret the interaction between face shape and gaze direction. These tests showed that dominance ratings were significantly higher for masculinised faces with direct gaze than for feminised faces with direct gaze $\left(F_{1,44}=7.78, p=0.008\right.$, partial $\left.\eta^{2}=0.15\right)$. By contrast, dominance ratings for masculinised and feminised faces with averted gaze did not differ significantly $\left(F_{1,44}<0.01, p=0.95\right.$, partial $\left.\eta^{2}<0.001\right)$. Additionally, these analyses revealed that dominance ratings were significantly higher for masculinised faces with direct gaze than 
for masculinised faces with averted gaze $\left(F_{1,44}=39.99, p<0.001\right.$, partial $\left.\eta^{2}=0.48\right)$ and were also significantly higher for feminised faces with direct gaze than for feminised faces with averted gaze $\left(F_{1,44}=16.98, p<0.001\right.$, partial $\left.\eta^{2}=0.28\right)$. $^{(1)}$

Collectively, these findings are consistent with our predictions that (i) sexual dimorphism of face shape would have a greater effect on the perceived dominance of faces with direct gaze than those with averted gaze and that (ii) gaze direction would have a greater effect on the perceived dominance of masculinised than feminised faces.

\section{Discussion}

We found that faces with direct gaze were generally rated as more dominant than those with averted gaze (see also Burgoon et al 1986). We also found that participants tended to rate masculinised faces as more dominant than feminised faces (see also DeBruine et al 2006; Perrett et al 1998), although this main effect of face shape only approached significance $(p=0.058)$. Perhaps more interestingly, we also found evidence that these effects of gaze direction and sexual dimorphism of 2-D face shape interact when judging the dominance of others.

Consistent with our prediction (i), we found that masculinised faces were rated as more dominant than feminised faces when they were shown with direct gaze, but not when they were shown with averted gaze. Consistent with our prediction (ii), we also found that the effect of gaze direction on perceptions of the dominance of others was significantly greater when participants judged the dominance of masculinised faces than when they judged the dominance of feminised faces, although both masculinised and feminised faces were rated as significantly more dominant when shown with direct gaze than when shown with averted gaze. These findings demonstrate that gaze direction and sexual dimorphism of 2-D face shape interact when judging the dominance of others. As we had predicted, participants appear to be particularly sensitive to shape cues associated with high dominance when rating the dominance of faces that are looking at them [prediction (i)] and particularly sensitive to gaze cues associated with dominance when rating the dominance of faces that possess physical cues associated with high dominance [prediction (ii)]. This interaction between gaze and shape cues may allow people to assess the dominance of others more efficiently than processing these social and physical cues independently would allow.

Our findings for integration of gaze direction and sexual dimorphism of 2-D face shape when perceiving the dominance of others complement previous findings for judgments of attractiveness, whereby attractiveness judgments were influenced by interactions between gaze direction and physical facial cues (eg attractive colour and texture cues-Jones et al 2006; sex of face judged-Conway et al 2008a; Mason et al 2005). Additionally, our findings complement previous studies showing that direct gaze increases attributions of anger to faces (Adams and Kleck 2005) and that viewers are more sensitive to sexually dimorphic cues when judging the sex of faces with direct gaze than when judging the sex of faces with averted gaze (Macrae et al 2002). Importantly, that direct gaze increases perceptions of both dominance (an antisocial trait) and attractiveness (a positive trait) indicates that gaze direction can have strikingly similar effects on

(1) A paired-samples $t$-test confirmed that the effect of gaze direction on the perceived dominance of masculinised faces (ie the mean dominance rating for masculinised faces with direct gaze minus the mean dominance rating for masculinised faces with averted gaze; mean difference $=1.01$, $\mathrm{SEM}=0.16$ ) and the effect of gaze direction on the perceived dominance of feminised faces (ie the mean dominance rating for feminised faces with direct gaze minus the mean dominance rating for feminised faces with averted gaze; mean difference $=0.71, \mathrm{SEM}=0.17$ ) differed significantly $\left(t_{44}=2.54, p=0.015\right.$, partial $\left.\eta^{2}=0.13\right)$. 
attributions of both positive and negative traits. Thus, whether direct gaze is perceived in a positive or negative manner appears to depend on the type of judgment made. Since attraction and dominance are both approach-oriented behaviours, that direct gaze has similar (positive) effects on both attributions of dominance and attractiveness is consistent with Adams and Kleck's (2005) finding that direct gaze increases attributions of approach-oriented emotions, regardless of whether they are positive (eg joy) or negative (eg anger). Our findings for gaze direction and dominance therefore support Adams and Kleck's proposal that perceptions of approach-oriented behaviours are increased by direct gaze. Additionally, our findings show that such effects are not limited to attributions of basic emotional states, but may also extend to attributions of approach-oriented social behaviours more generally.

When rating men and women for sex-stereotyped traits (eg height) with Likerttype scales, raters may anchor their ratings differently for each sex by using sex-specific referents (eg Biernat et al 1991). For example, the large sex difference observed when height estimates are given in inches is not apparent when subjects are asked to rate the height of men and women with Likert-type scales, implicating top-down processes in how stereotyped mental representations are expressed on subjectively defined rating scales (Biernat et al 1991). In our experiment, however, the significant main effect of sex of face judged indicated that men were rated as significantly more dominant than women. This finding suggests that the stereotypically masculine trait of dominance is not rated relative to sex-specific referents in the way that height, for example, appears to be. While men received higher dominance ratings than women in our study, this sex difference in dominance ratings may, of course, be more pronounced if a more objective rating scale were used, potentially bringing our findings more in line with Biernat et al's (1991) suggestion.

While our findings suggest that gaze direction and face shape interact when perceiving the dominance of others, the mechanisms and processes that underpin this integration remain unclear. In particular, further research is needed to establish whether the integration of social information from gaze direction and sexually dimorphic 2-D face cues reflects low-level biases in the analysis of different face patterns (ie is driven primarily by simple physical properties of the stimulus), more high-level cognitive biases (ie deliberate inferences about the likely attitudes and intentions of others), or a combination of these factors. Further research is also needed to investigate how explicit social knowledge about familiar individuals influences how gaze and face shape are integrated when judging dominance. While our findings suggest that gaze direction and face shape interact when judging the dominance of unfamiliar individuals, this interaction may not necessarily occur when judging familiar individuals. Indeed, examining whether explicit social knowledge of the target qualifies the interaction observed for familiar faces in our experiment may shed light on the extent to which deliberate inferences about the likely attitudes and intentions of others contribute to these effects.

We show that the effects of gaze direction and sexual dimorphism of 2-D face shape interact when perceiving the dominance of others. Combining information from multiple cues of dominance (eg gaze direction, face shape) may function to increase the efficiency with which people perceive the dominance of others, promoting fluent social interactions. Additionally, our findings present novel evidence for complex integration of invariant physical cues and changeable social cues in face perception. Such interactions are a specific prediction of recent models of face processing that have emphasised interactions between different cues when processing faces (eg Calder and Young 2005; Haxby et al 2000), and problematic for alternative models in which functionally distinct facial characteristics are thought to be processed independently (Bruce and Young 1986). 


\section{References}

Adams R B Jr, Kleck R E, 2003 "Perceived gaze direction and the processing of facial displays of emotion" Psychological Science $141644-647$

Adams R B Jr, Kleck R E, 2005 "Effects of direct and averted gaze on the perception of facially communicated emotion" Emotion 5 3-11

Biernat M, Manis M, Nelson T E, 1991 "Stereotypes and standards of judgment" Journal of Personality and Social Psychology $60485-499$

Bindemann M, Burton A M, Langton S R H, 2008 "How do eye-gaze and facial expression interact?" Visual Cognition $16708-733$

Bruce V, Young A W, 1986 "Understanding face recognition" British Journal of Psychology 77 $305-327$

Buckingham G, DeBruine L M, Little A C, Welling L L M, Conway C A, Tiddeman B P, Jones B C, 2006 "Visual adaptation to masculine and feminine faces influences generalized preferences and perceptions of trustworthiness" Evolution and Human Behavior 27381 - 389

Burgoon J K, Coker D A, Coker R A, 1986 "Communicative effects of gaze behavior" Human Communication Research 12 495-524

Calder A J, Young A W, 2005 "Understanding facial identity and facial expression recognition" Nature Reviews Neuroscience $6641-651$

Conway C A, Jones B C, DeBruine L M, Little A C, 2008a "Evidence for adaptive design in human gaze preference" Proceedings of the Royal Society of London, Series B 275 63-69

Conway C A, Jones B C, DeBruine L M, Little A C, 2009 "Sexual dimorphism of male face shape, partnership status and the temporal context of relationship sought modulate women's preferences for direct gaze" British Journal of Psychology, in press; doi:10.1348/000712609X436435

Conway C A, Jones B C, DeBruine L M, Little A C, Hay J, Perrett D I, Feinberg D R, 2008b "Integrating physical and social cues when forming face preferences: Differences among low and high anxiety individuals" Social Neuroscience 3 89-95

Deaner R O, Shepherd S V, Platt M L, 2007 "Familiarity accentuates gaze cueing in women but not men" Biology Letters 3 64-67

DeBruine L M, Jones B C, Little A C, Boothroyd L G, Perrett D I, Penton-Voak I S, Cooper P A, Penke L, Feinberg D R, Tiddeman B P, 2006 "Correlated preferences for facial masculinity and ideal or actual partner's masculinity" Proceedings of the Royal Society of London, Series B $2731355-1360$

Emery N J, 2000 "The eyes have it: the neuroethology, function and evolution of social gaze" Neuroscience and Biobehavioral Reviews $24581-604$

Feinberg D R, Jones B C, Little A C, Burt D M, Perrett D I, 2005 "Manipulation of fundamental and formant frequencies influence the attractiveness of human male voices" Animal Behaviour $69561-568$

Fink B, Neave N, Seydel H, 2007 "Male facial appearance signals physical strength to women" American Journal of Human Biology $1982-87$

Fink B, Penton-Voak I S, 2002 "Evolutionary psychology of facial attractiveness" Current Directions in Psychological Sciences 11 154-158

Haxby J V, Hoffman E A, Gobbini M I, 2000 "The distributed human neural system for face perception" Trends in Cognitive Sciences $4223-233$

Hess U, Blairy S, Kleck R E, 2000 "The influence of facial emotion displays, gender, and ethnicity on judgments of dominance and affiliation" Journal of Nonverbal Behavior 24 265-283

Jones B C, Little A C, Burt D M, Perrett D I, 2004 "When facial attractiveness is only skin deep" Perception $33569-576$

Jones B C, Perrett D I, Little A C, Boothroyd L G, Cornwell R E, Feinberg D R, Tiddeman B P, Whiten S, Pitman R M, Hillier S G, Burt D M, Stirrat M R, Law Smith M J, Moore F R, 2005 "Menstrual cycle, pregnancy and oral contraceptive use alter attraction to apparent health in faces" Proceedings of the Royal Society of London, Series B 272347 - 354

Jones B C, DeBruine L M, Little A C, Conway C, Feinberg D R, 2006 "Integrating gaze direction and expression in preferences for attractive faces" Psychological Science $17588-591$

Jones B C, DeBruine L M, Little A C, Conway C A, Welling L L M, Smith F G, 2007 "Sensation seeking and men's face preferences" Evolution and Human Behavior 28439 - 446

Kampe K K, Frith C D, Dolan R J, Frith U, 2001 "Reward value of attractiveness and gaze" Nature 413589

Kraut R, Olson J, Banaji M, Bruckman A, Cohen J, Couper M, 2004 "Psychological research online: Report of Board of Scientific Affairs' Advisory Group on the conduct of research on the Internet" American Psychologist 59105 - 177 
Little A C, Burt D M, Penton-Voak I S, Perrett D I, 2001 "Self-perceived attractiveness influences human female preferences for sexual dimorphism and symmetry in male faces" Proceedings of the Royal Society of London, Series B $26839-44$

Little A C, Jones B C, Penton-Voak I S, Burt D M, Perrett D I, 2002 "Partnership status and the temporal context of relationships influence human female preferences for sexual dimorphism in male face shape" Proceedings of the Royal Society of London, Series B 269 1095-1100

Macrae C N, Hood B M, Milne A B, Rowe A C, Mason M F, 2002 "Are you looking at me? Eye gaze and person perception" Psychological Science $13460-464$

Mason M F, Tatkow E P, Macrae C N, 2005 "The look of love: gaze shifts and person perception" Psychological Science $16236-239$

Oosterhof N N, Todorov A, 2008 "The functional basis of face evaluation" Proceedings of the National Academy of Sciences of the USA $10511087-11092$

Penton-Voak I S, Little A C, Jones B C, Burt D M, Perrett D I, 2003 "Measures of human female condition predict preferences for sexually dimorphic characteristics in men's faces" Journal of Comparative Psychology $117264-271$

Penton-Voak I S, Perrett D I, Castles D L, Kobayashi T, Burt D M, Murray L K, Minamisawa R, 1999 "Menstrual cycle alters face preference" Nature 399741 - 742

Perrett D I, Lee K J, Penton-Voak I S, Rowland D R, Yoshikawa S, Burt D M, Henzi S P, Castles D L, Akamatsu S, 1998 "Effects of sexual dimorphism on facial attractiveness" Nature 394 884-887

Perrett D I, Penton-Voak I S, Little A C, Tiddeman B P, Burt D M, Schmidt N, Oxley R, Kinloch N, Barrett L, 2002 "Facial attractiveness judgments reflect learning of parental age characteristics" Proceedings of the Royal Society of London, Series B 269873 - 880

Puts D A, Gaulin S J C, Verdolini K, 2006 "Dominance and the evolution of sexual dimorphism in human voice pitch" Evolution and Human Behavior 27 283-296

Rhodes G, 2006 "The evolutionary psychology of facial beauty" Annual Review of Psychology 57 $199-226$

Tiddeman B P, Perrett D I, Burt D M, 2001 "Prototyping and transforming facial textures for perception research" IEEE Computer Graphics and Applications, Research $2142-50$

Welling L L M, Jones B C, DeBruine L M, Conway C A, Law Smith M J, Little A C, Feinberg D R, Sharp M A, Al-Dujaili E A S, 2007 "Raised salivary testosterone in women is associated with increased attraction to masculine faces" Hormones and Behavior 52 156-161

Welling L L M, Jones B C, DeBruine L M, 2008 “Sex drive is positively associated with women's preferences for sexual dimorphism in men's and women's faces" Personality and Individual Differences 44161 - 170

Wilson M, Daly M, 2004 "Do pretty women inspire men to discount the future?" Biology Letters 271 S177-S179 


\section{PERTEPTION}

VOLUME 382009

www.perceptionweb.com

Conditions of use. This article may be downloaded from the Perception website for personal research by members of subscribing organisations. Authors are entitled to distribute their own article (in printed form or by e-mail) to up to 50 people. This PDF may not be placed on any website (or other online distribution system) without permission of the publisher. 